\title{
MODIS On-Orbit Thermal Emissive Bands Lifetime Performance
}

\author{
Sriharsha Madhavan ${ }^{1}$, Aisheng $\mathrm{Wu}^{1}, \mathrm{Na}_{\text {Chen }}{ }^{1}$, and Xiaoxiong Xiong ${ }^{2}$ \\ ${ }^{1}$ Science Systems and Applications Inc., 10210, Greenbelt Road, Lanham, MD 20706 \\ ${ }^{2}$ Sciences and Exploration Directorate, NASA/GSFC, Greenbelt, MD 20771
}

\begin{abstract}
MODerate resolution Imaging Spectroradiometer (MODIS), a leading heritage sensor in the fleet of Earth Observing System for the National Aeronautics and Space Administration (NASA) is in space orbit on two spacecrafts. They are the Terra (T) and Aqua (A) platforms. Both instruments have successfully continued to operate beyond the 6 year design life time, with the T-MODIS currently functional beyond 15 years and the A-MODIS operating beyond 13 years respectively. The MODIS sensor characteristics include a spectral coverage from $0.41 \mu \mathrm{m}-14.4 \mu \mathrm{m}$, of which wavelengths ranging from $3.7 \mu \mathrm{m}-14.4 \mu \mathrm{m}$ cover the thermal infrared region also referred to as the Thermal Emissive Bands (TEBs). The TEBs is calibrated using a v-grooved BlackBody (BB) whose temperature measurements are traceable to the National Institute of Standards and Technology temperature scales. The TEBs calibration based on the onboard BB is extremely important for its high radiometric fidelity. In this paper, we provide a complete characterization of the lifetime instrument performance of both MODIS instruments in terms of the sensor gain, the Noise Equivalent difference Temperature, key instrument telemetry such as the BB lifetime trends, the instrument temperature trends, the Cold Focal Plane telemetry and finally, the total assessed calibration uncertainty of the TEBs.
\end{abstract}

Keywords: MODIS, Terra, Aqua, Thermal Emissive bands, Blackbody, Calibration

\section{Introduction}

The MODerate-resolution Imaging Spectroradiometer (MODIS) is a legacy space borne sensor in the fleet of Earth Observing System (EOS) for the National Aeronautics and Space Administration (NASA). The instrument is in space orbit on two spacecrafts, namely the Terra (T) and Aqua (A) platforms [1-4]. The T-platform is on a sun synchronous morning orbit while the A-platform is on a similar sun synchronous afternoon orbit. They have an equatorial crossing time of approximately 10:30 AM and 1:30 PM local time, respectively. Hence, the Earth is covered by both MODIS instruments within a day, which has significantly helped the scientific community to capture any observable diurnal changes in the Earth's climate. MODIS is a paddle broom scanning radiometer that includes a $360^{\circ}$ rotating double-sided scan mirror and several on-board calibrators (OBCs) as illustrated in Figure 1. The MODIS instruments remotely capture the top of atmosphere (TOA) radiance from the Earth in 36 spectral bands varying from $0.41 \mu \mathrm{m}$ 
to $14.4 \mu \mathrm{m}$, at three different geospatial resolutions of $250 \mathrm{~m}, 500 \mathrm{~m}$, and $1 \mathrm{~km}$, respectively. The spectral wavelengths long ward of $3.7 \mu \mathrm{m}$ covers the thermal infrared region which is split into 16 Thermal Emissive Bands (TEBs). The 36 spectral bands are located on four Focal Plane Assemblies (FPAs) that includes the visible FPA, the near infrared FPA, the Short and Middle wave InfraRed (SMIR) FPA, and the Long Wave InfraRed (LWIR) FPA. The SMIR and LWIR FPAs house the thermal sensors that are maintained at a nominally cooled temperature of approximately $83 \mathrm{~K}$. Figure 2 shows the four FPAs along with the detector layout for each of the MODIS bands, the red outline highlighting the cold FPAs, including all the TEB detectors. The TEB detectors are calibrated using a well referenced source that comprises of a v-grooved BlackBody (BB). Figure 1 shows the setup of the various OBCs, and in particular the BB in highlight is used for the detector calibration for each of the TEBs [5]. The TEB calibration is a two point calibration with the $\mathrm{BB}$ raw counts providing the calibrating signal at a nominal temperature while the Space-View (SV) signal is used to determine the background signal digital number (DN) that would help correct the electronic offsets and thermal background from the actual measurements [6]. Table 1 gives the key design parameters including the calibration requirements for the detector noise and the primary application for each of the TEBs.

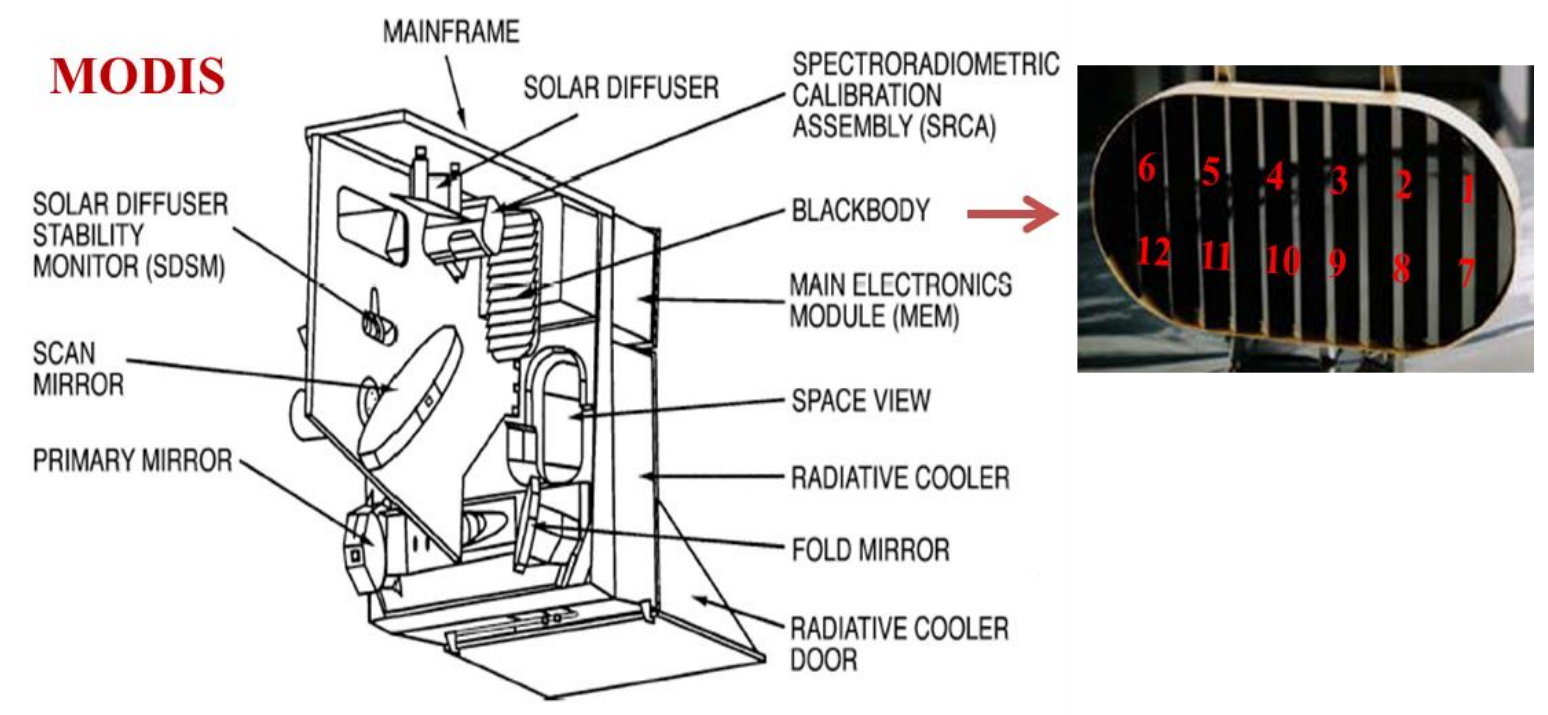

Figure 1. MODIS Instrument setup with On-board Calibrators.

With the given instrument background, the rest of the paper is organized as follows. Section 2 briefly goes over the fundamental calibration equation and the methodology. This is followed by the TEB performance which covers the following subsetted aspects. The subsections include the long term instrument gain and noise characterization, various instrument related telemetry and the assessed calibration uncertainty over the lifetime. Finally, the paper is closed with an overall synopsis of the lifetime TEB performance for both T- and A-MODIS respectively. 


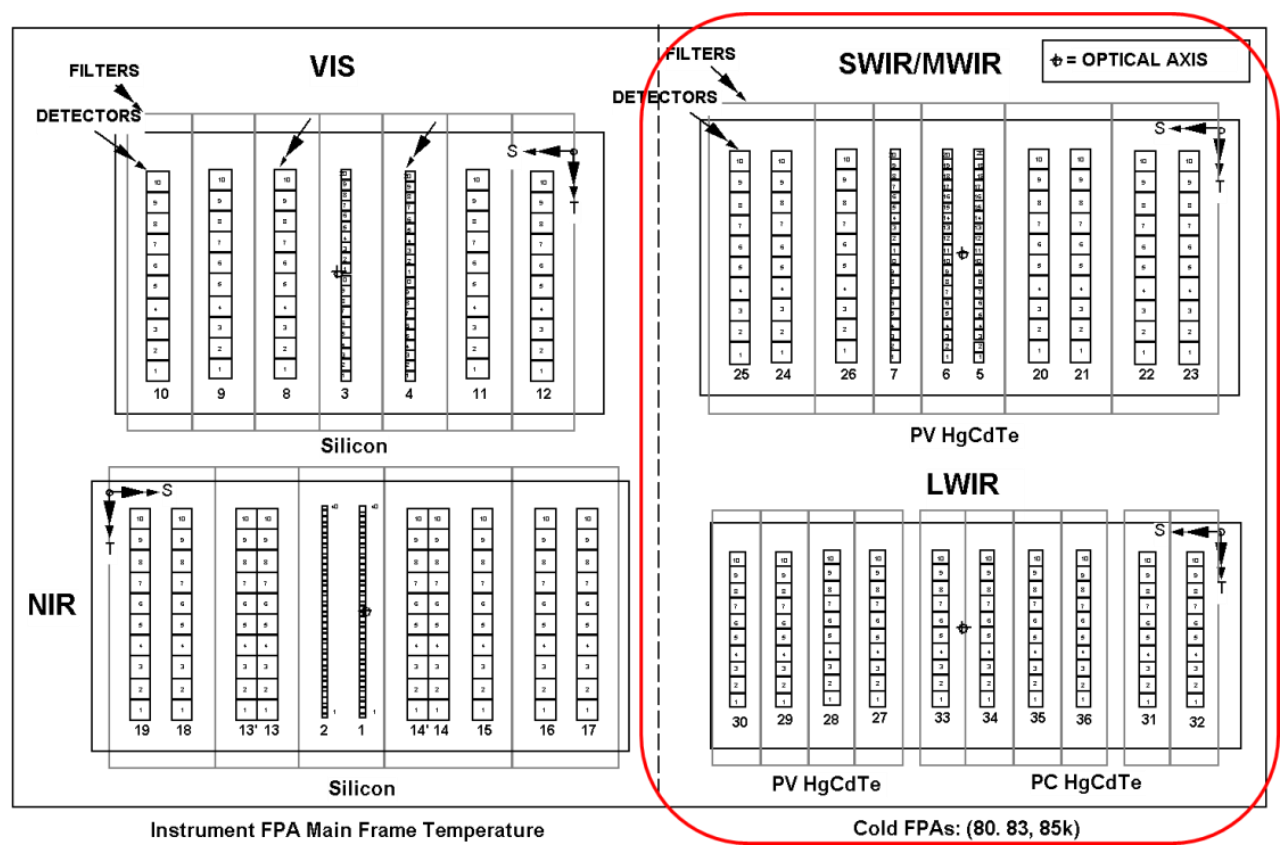

Figure 2. MODIS Focal Plane layout (red box highlighting the TEB).

Table 1. MODIS spectral band design specifications. CW is center wavelength in $\mu \mathrm{m}$; $\mathrm{BW}$ is bandwidth in $\mu \mathrm{m}$; Ttyp is typical temperature in ${ }^{\circ} \mathrm{K}$; NEdT is noise equivalent difference temperature in ${ }^{\circ} \mathrm{K}$.

\begin{tabular}{llllll}
\hline TEB Band & CW & BW & Ttyp & NEdT & Primary Use \\
\hline 20 & 3.75 & 0.18 & 300 & 0.05 & \\
21 & 3.96 & 0.06 & 335 & 0.20 & Surface/cloud temperature \\
22 & 3.96 & 0.06 & 300 & 0.07 & \\
23 & 4.05 & 0.06 & 300 & 0.07 & \\
\hline 24 & 4.47 & 0.07 & 250 & 0.25 & \multirow{2}{*}{ Atmospheric temperature } \\
25 & 4.52 & 0.07 & 275 & 0.25 & \\
\hline 27 & 6.72 & 0.36 & 240 & 0.25 & \multirow{2}{*}{ Water vapor } \\
28 & 7.33 & 0.30 & 250 & 0.25 & \\
\hline 29 & 8.55 & 0.30 & 300 & 0.05 & Cloud properties \\
\hline 30 & 9.73 & 0.30 & 250 & 0.25 & Ozone \\
\hline 31 & 11.03 & 0.50 & 300 & 0.05 & \multirow{2}{*}{ Surface/cloud temperature } \\
32 & 12.02 & 0.50 & 300 & 0.05 & \\
\hline 33 & 13.34 & 0.30 & 260 & 0.25 & \\
34 & 13.64 & 0.30 & 250 & 0.25 & \multirow{2}{*}{ Cloud top altitude } \\
35 & 13.94 & 0.30 & 240 & 0.25 & \\
36 & 14.24 & 0.30 & 220 & 0.35 & \\
\hline
\end{tabular}




\section{TEB Calibration}

The TEB calibration is based on a quadratic algorithm that converts the digital response of the sensor to it's at sensor aperture radiance. In order to perform the conversion the digital response is first corrected for background and optical crosstalk (crosstalk correction only applied to TMODIS PC bands B32-36) which is represented in digital counts known as dn. The second step is to compute the calibration linear coefficient $b_{1}$ using the calibrator radiance ( $\mathrm{L}_{\mathrm{CAL}}$ ) as observed

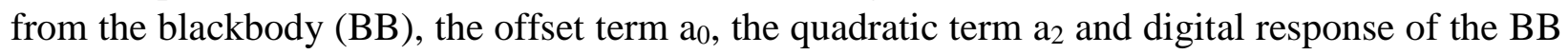
given in $\mathrm{dn}_{\mathrm{BB}}$. Equations (1) and (2) summarize the calibration operation performed on all TEB channels on a scan by scan basis. The calibration terms $\mathrm{a}_{0}$ and $\mathrm{a}_{2}$ shown in equation (2) are computed on a quarterly basis during the BB warm-up/cool-down (WUCD) process. Details on the various calibration parameters shown in equation (1) and (2) can be found in [7].

$$
\begin{gathered}
L_{C A L}=R V S_{B B} \varepsilon_{B B} L_{B B}+\left(R V S_{S V}-R V S_{B B}\right) L_{S M}+R V S_{B B}\left(1-\varepsilon_{B B}\right) \varepsilon_{c a v} L_{c a v} \\
b_{1}=\left(L_{C A L}-a_{0}-a_{2} d n_{B B}^{2}\right) / d n_{B B}
\end{gathered}
$$

In the above calibration equations, the terms RVS, $\varepsilon$, are determined at pre-launch whereas the $\mathrm{a}_{0}, \mathrm{a}_{2}$, and the Band $21 \mathrm{~b}_{1}$ coefficients are updated on a quarterly frequency (if necessary), using on-board BB WUCD measurements. The strategy for these updates in both Version (V) 5 and 6 are summarized in Table 2.

\begin{tabular}{|c|c|c|}
\hline Terra & V5 & Changes in V6 \\
\hline B20,22-32 & Warm-up a0/a2 & $\mathrm{a} 0=0$, cool-down a2 \\
\hline B21 & $\mathrm{a} 0=0$ and $\mathrm{a} 2=0$ & No change \\
\hline B33-36 & $\mathrm{a} 0=0$, Warm-up a2 & $\mathrm{a} 0=0$, cool-down a2 \\
\hline Aqua & V5 & Changes in V6 \\
\hline $\mathrm{B} 20,22-30$ & $\mathrm{PL} \mathrm{a0/a2}$ & Adjust PL a2 based on cool-down \\
\hline B21 & $\mathrm{a} 0=0$ and $\mathrm{a} 2=0$ & No change \\
\hline B31-32 & Warm-up a0/a2 & $\mathrm{a} 0=0$, cool-down a 2 \\
\hline B33-36 & $\mathrm{a} 0=0, \mathrm{PL} a 2$ & Adjust PL a 2 based on cool-down \\
\hline
\end{tabular}

Table 2. TEB $\mathrm{a}_{0}, \mathrm{a}_{2}$ update strategy. 


\section{TEB Performance}

Three key aspects of the MODIS TEB performance are discussed. These include the instrument gain for all bands (also referred to as the reciprocal of the linear calibration term $b_{1}$ ), associated sensor noise characterized in terms of the Noise Equivalent difference Temperature (NEdT) [8], various instrument telemetries such as the $\mathrm{BB}$, instrument, and focal plane temperature trends, and lastly the instrument assessed calibration uncertainty.

\subsection{Sensor Gain and Noise}

Based on the long term gain trends for both T-MODIS and A-MODIS as shown in Figure 3, a more or less stable detector response is noticed for the SMIR bands excluding sensor configuration changes and instrument reset events. However, in the case of T-MODIS PV LWIR bands (27-30), large downward drifts in gain are observe which have been identified to be due to the electronic crosstalk contamination [9]. The PC LWIR bands of T-MODIS are relatively stable with maximum changes in the order of approximately $2 \%$ over lifetime. In contrast, AMODIS has exhibited stable long term variations for the PV LWIR bands. However, due to the loss of cold focal plane cooler margin [10], the instrument response for the PC LWIR bands has caused significant oscillations due to the seasonal changes in the cold focal plane temperatures.

In order to highlight the differences in the noise performance for T- and A-MODIS, the lifetime normalized NEdT (weekly sampled, scan averaged) trends for good detectors are provided. Figure 4 gives the normalized NEdT trend for both T-MODIS and A-MODIS respectively. The trend clearly shows that these detectors meet the design specifications for the almost entirety of their mission. It is noted that the noisy nature for T-MODIS PV LWIR bands have increased since 2010, is chiefly attributed to the severe degradation in the electronic circuitry of these bands due to electronic crosstalk. In comparison, the normalized band averaged NEdT trends for A-MODIS clearly meet the noise requirements; furthermore, the noise levels are lower by at least a factor of 2 in comparison to the normalized specification line of 1.0. 

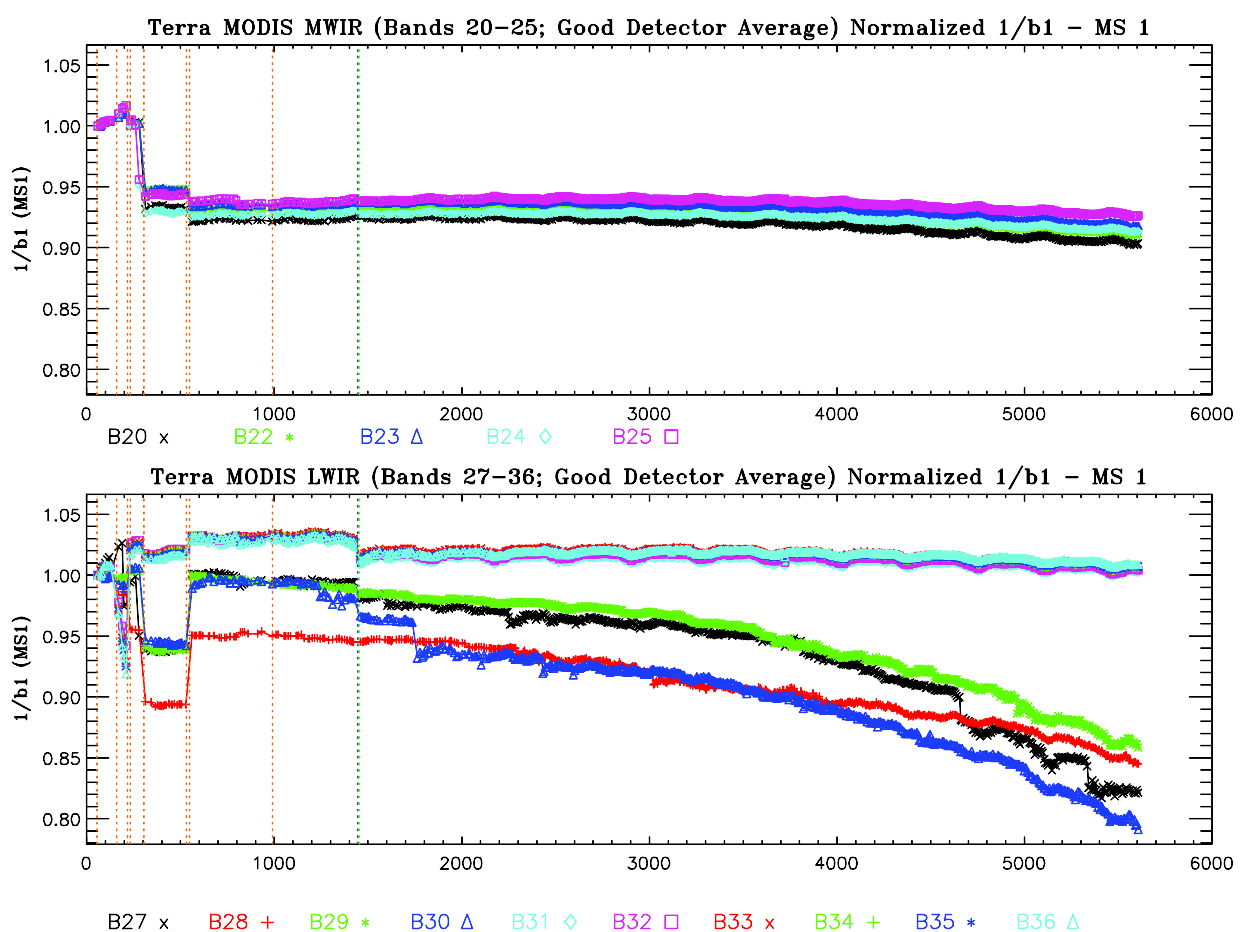

a.
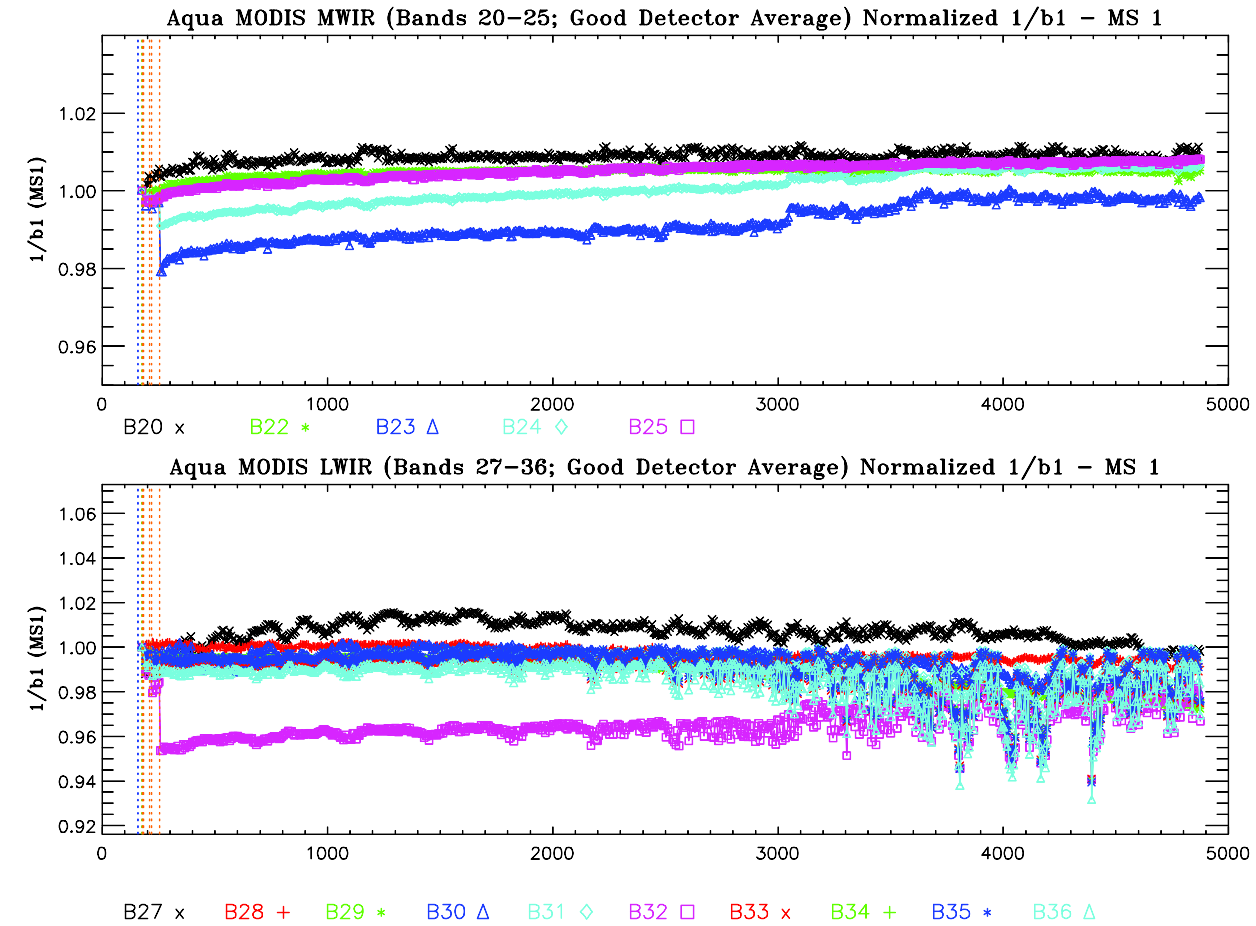

b.

Figure 3. Long-term gain (1/b1) trending for TEBs of a. T-MODIS b. A-MODIS. 

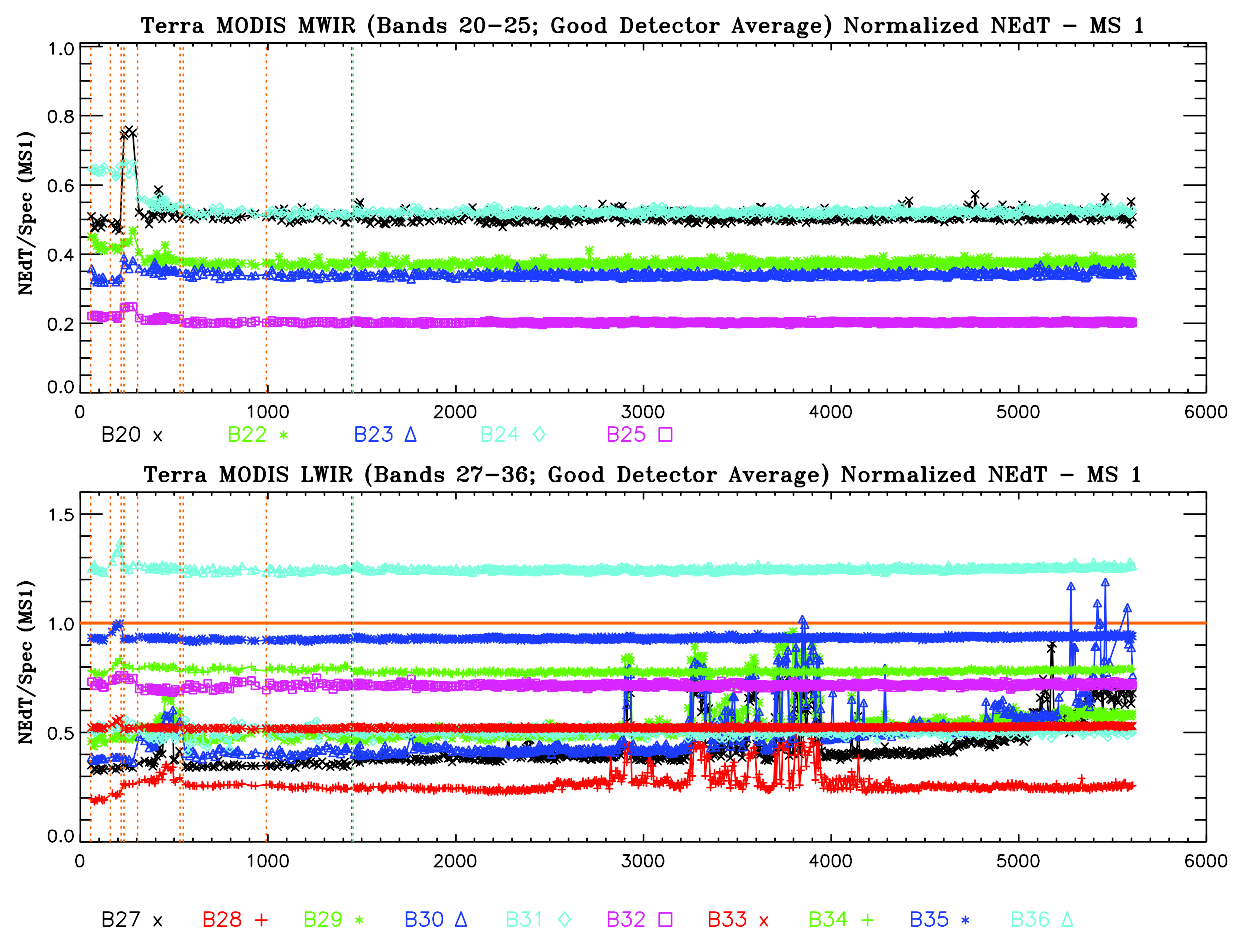

a.
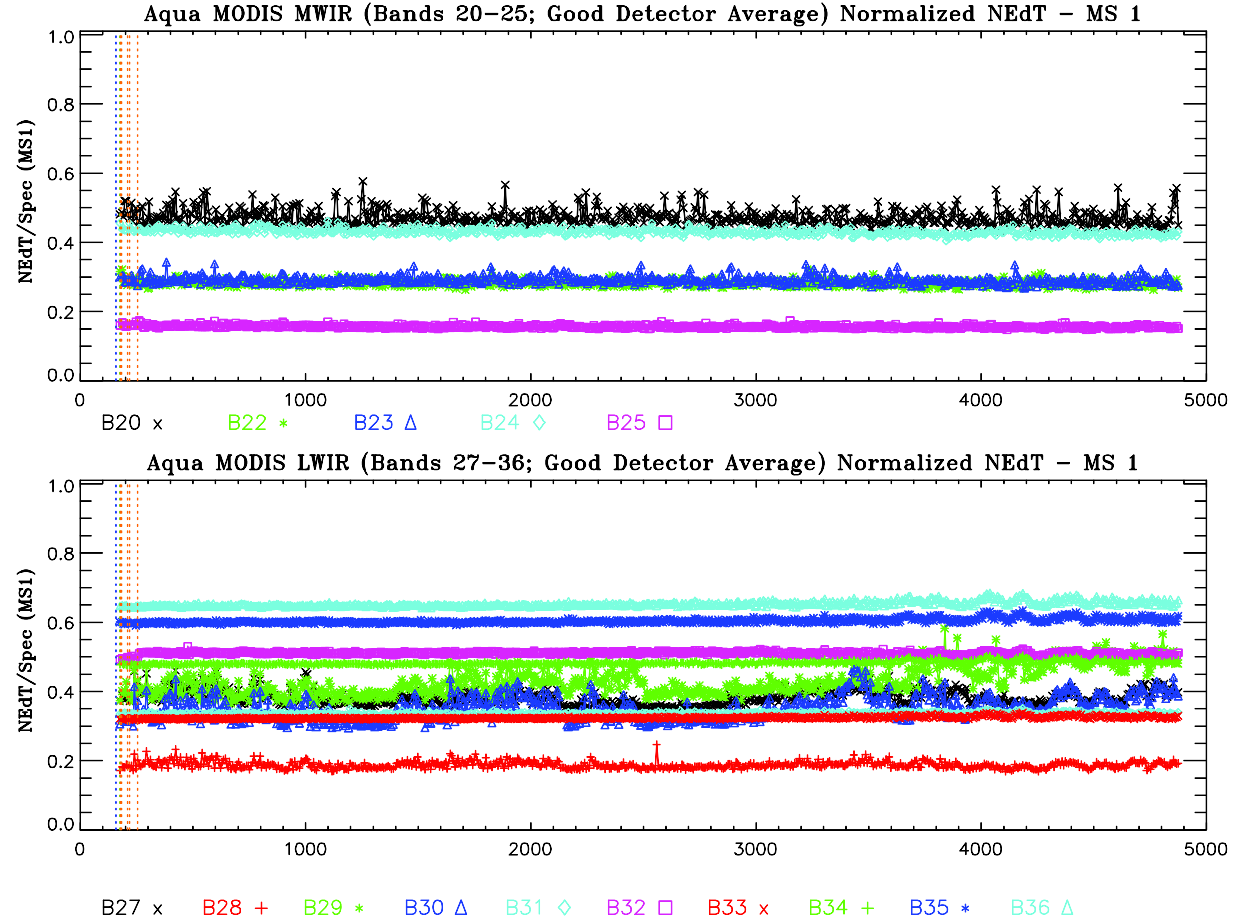

b.

Figure 4. Long-term normalized NEdT trending for TEBs of a. T-MODIS b. A-MODIS. 


\subsection{Instrument related Telemetry}

For T-MODIS the BB temperature is nominally controlled at $290 \mathrm{~K}$ whereas A-MODIS is maintained at $285 \mathrm{~K}$. Figures 5a. and 5b. shows the lifetime trends of the BB temperatures for both T- and A-MODIS at their set temperatures. The data presented is a weekly average of the 12 thermistors. Based on the lifetime trends, the variability of the BB temperatures is assessed to be within $15 \mathrm{mK}$ for T-MODIS and within $5 \mathrm{mK}$ for A-MODIS. Similarly, the instrument temperatures for T-MODIS (Figure 5c.) have gone up by $3.5 \mathrm{~K}$ over 15 years, while the instrument temperature has increased by about $2 \mathrm{~K}$ for A-MODIS (Figure 5d.). Based on Figure 5e., the cold focal plane temperatures have essentially been constant for T-MODIS barring early mission. For A-MODIS as mentioned earlier the loss of cooler margin has caused the focal plane temperature (Figure 5f.) to oscillate within $0.4 \mathrm{~K}$ since 2010. Currently, the cold focal plane seems to have marginally stabilized and is about $83.1 \mathrm{~K}$ with $\pm 0.1 \mathrm{~K}$ seasonal changes.

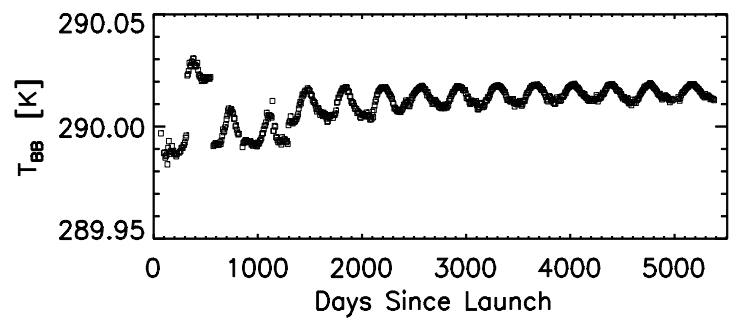

a.

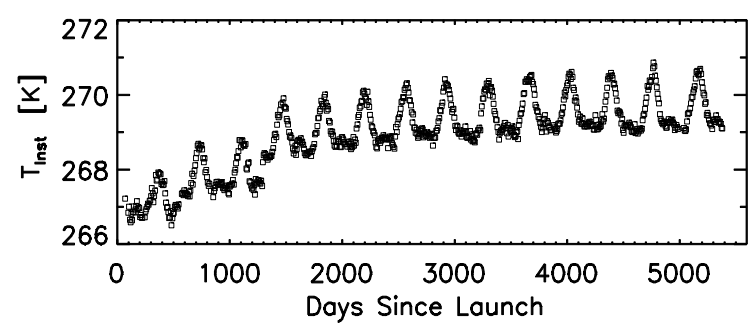

c.

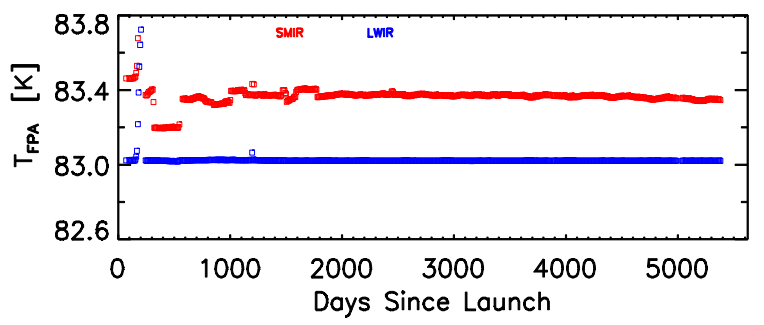

e.

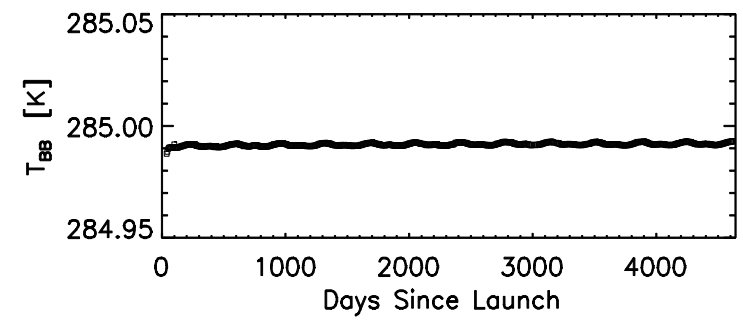

b.

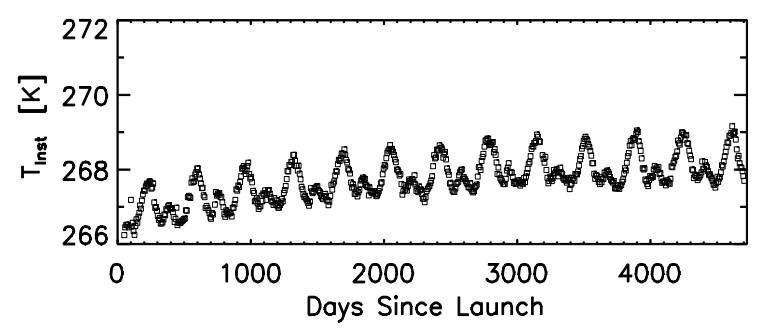

d.

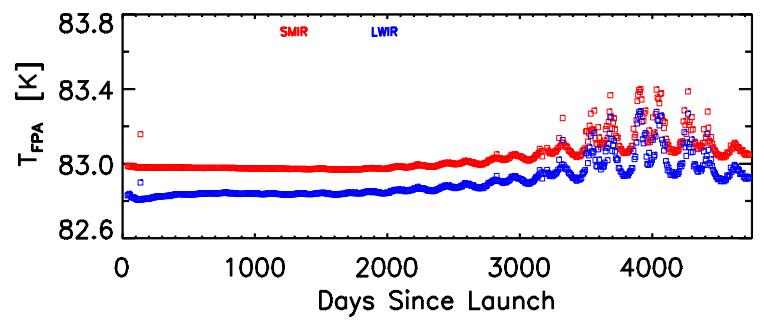

f.

Figure 5. Long-term telemetry (BB, Instrument, Focal Plane) trending for TEBs of T-MODIS (a.,c.,e.) and AMODIS (b.,d.,f.) 


\subsection{Calibration Uncertainty}

The total TEB uncertainty is expressed as root sum of squares of each individual uncertainty term. The uncertainty term in principle is evaluated via a partial derivative of the Earth view retrieval equation with respect to each individual calibration term. Details on the mechanism can be found in [7]. Figure 6 shows the bar chart of the assessed uncertainties for the TEBs of T-and A- MODIS evaluated at different years. Also, these are evaluated for typical scene radiance levels and close to 'at nadir' acquisitions. Based on the charts shown, the uncertainties assessed for T-MODIS band 30 currently exceeds the design requirement of $1 \%$. As aforementioned, the electronic crosstalk impact has severely impacted the calibration terms $a_{0}, a_{2}$ and $b_{1}$ due to which the uncertainties have significantly increased. Apart from that most of the other bands meet the design requirements with the exception of band 36. It was assessed at pre-launch that all detectors violated the uncertainty requirements and have behaved more or less in a similar fashion on-orbit. For A-MODIS, the TEBs have met the required calibration uncertainty since launch to date.

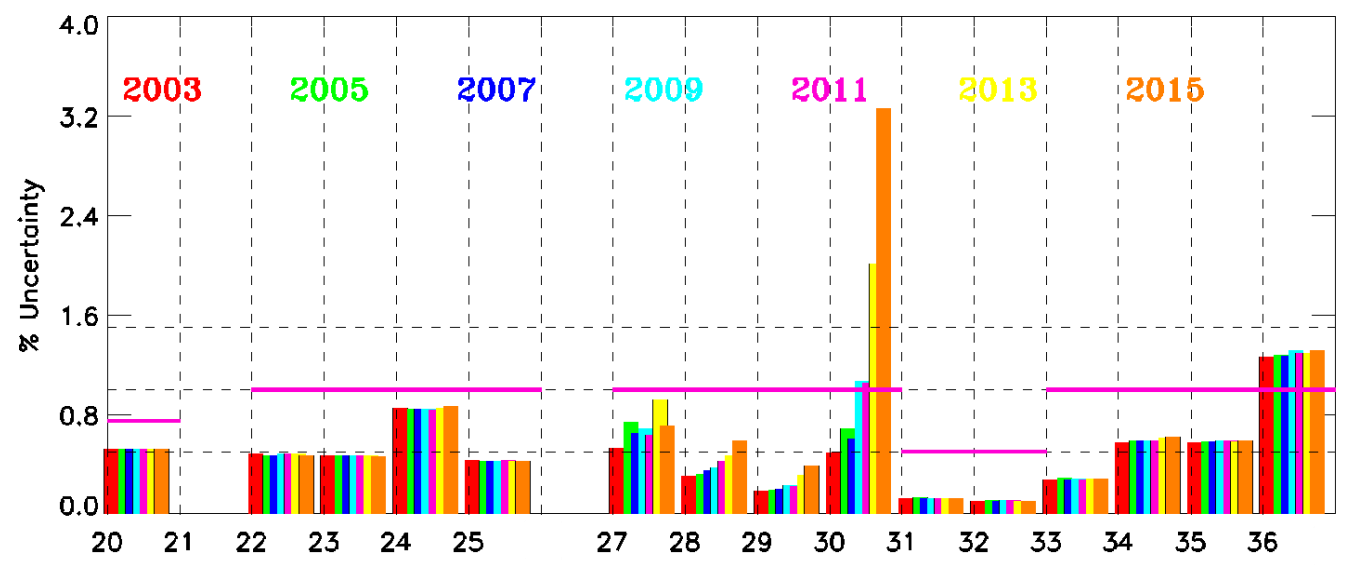

a.

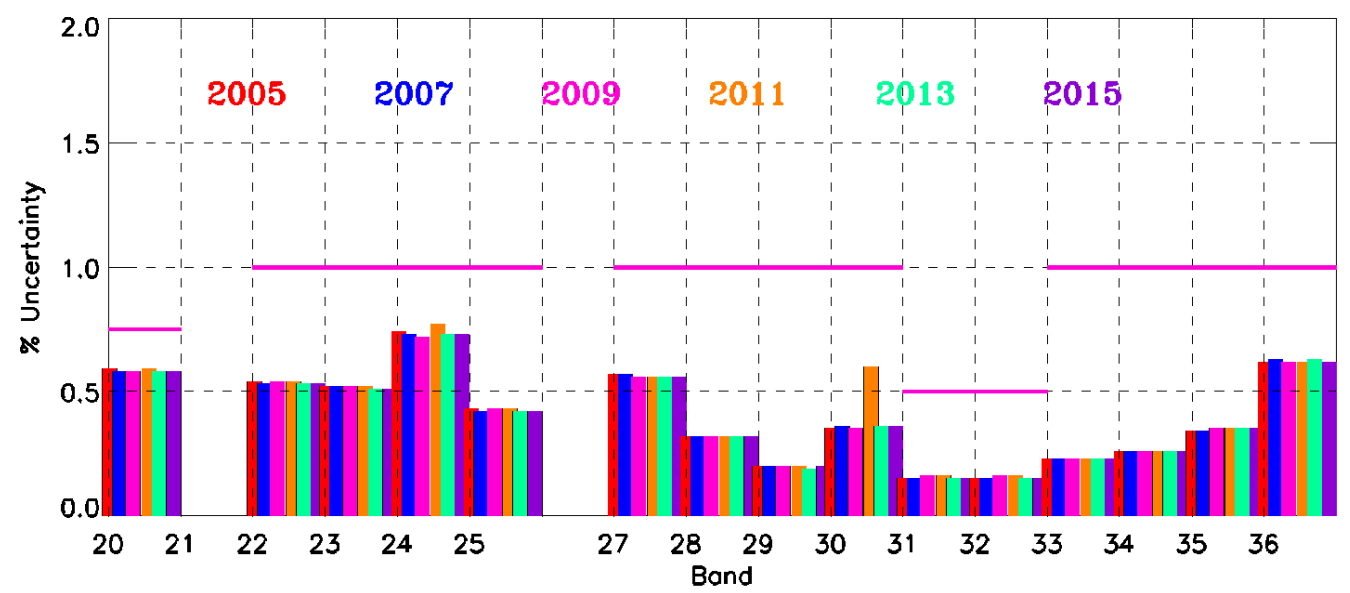

b.

Figure 6. Uncertainty bar-chart for TEBs of a. T-MODIS, b. A-MODIS 


\section{Summary}

MODIS a key legacy sensor on the T- and A- platforms has successfully operated in space far exceeding their individual design lifetime of 6 years. Both sensors have exhibited very stable performance in terms of the instrument and BB temperatures. The cold focal plane of A-MODIS was found to have an issue of maintaining its set point of $83 \mathrm{~K}$ starting 2007 and had reached a high point of close to $83.4 \mathrm{~K}$ by 2011 2012. Thereafter a marginal return of stability in the LWIR cooler margin has been assessed. The impact on the calibration due to the loss of cooler margin is found to be minimal since the TEB calibration is based on a scan-by-scan basis. A majority of the impact is captured by the linear calibration term. For T-MODIS the cold focal plane has been essentially constant throughout the lifetime. In terms of sensor performance AMODIS has exhibited very slow changes in terms of gain and NEdT. However, in the case of TMODIS the PV LWIR bands have shown large downward drifts in instrument gain, with correlating increase in the NEdT since 2010. Electronic crosstalk contamination in these bands has been identified to be the root cause. Finally, the assessed TEB uncertainties corroborate the sensor performance, have found to be meeting the design requirements barring the exceptions noted earlier. Thus, an accurate sensor calibration is extremely important to achieve high radiometric fidelity as in the case of MODIS, which in turn allows high quality geophysical retrievals that are impactful for various climate and environmental studies.

\section{Acknowledgements}

The authors would like to thank all current and past members of the MCST for their many and varied contributions over the years. In addition thanks go to the science team members we have collaborated with, leading to greater understanding of instrument performance, impacts on the science products and improvements in our calibration methodologies.

\section{References}

[1] Esaias, W. E., M. R. Abbott, I. Barton, O. W. Brown, J. W. Campbell, K. L. Carder, D. K. Clark, R. L. Evans, F. E. Hoge, H. R. Gordon, W. P. Balch, R. Letelier, and P. J. Minnett, "An Overview of MODIS Capabilities for Ocean Science Observations," IEEE Trans. Geosci. Remote Sensing, 36, 1250-1265 (1998).

[2] Justice, C. O., E. Vermote, J. R. G. Townshend, R. Defries, D. P. Roy, D. K. Hall, V. V. Salomonson, J. L. Privette, G. Riggs, A. Strahler, W. Lucht, R. B. Myneni, P. Lewis, and M. J. Barnsley, "The Moderate Resolution Imaging Spectroradiometer (MODIS): Land Remote Sensing for Global Change Research," IEEE Trans. Geosci. Remote Sensing, 36, 1228-1249 (1998).

[3] King, M. D., W. P. Menzel, Y. J. Kaufman, D. Tanre, B. C. Gao, S. Platnick, S. A. Ackerman, L. A. Remer, R. Pincus, and P. A. Hubanks, "Cloud and Aerosol Properties, Precipitable Water, and Profiles of Temperature and Water Vapor from MODIS," IEEE Trans. Geosci. Remote Sensing, 41, 442-458 (2003).

[4] Parkinson, C. L., "Summarizing the first ten years of NASA's Aqua mission," IEEE Journal of Selected Topics in Applied Earth Observations and Remote Sensing, 6(3), 1179-1188 (2013). 
[5] Xiong, X., K. Chiang, A. Wu, W. Barnes, B. Guenther, and V. Salomonson, "Multiyear on-orbit calibration and performance of Terra MODIS thermal emissive bands," IEEE Trans. Geosci. Remote Sens. 46, 1790-1803 (2008).

[6] Xiong, X., B. Wenny, A. Wu, and W. Barnes, "MODIS On-board Blackbody Function and Performance", IEEE Trans. Geosci. Remote Sens. 47, (2009).

[7] Xiong, X., A. Wu, B. N. Wenny, S. Madhavan, Z. Wang, Y. Li, N. Chen, W. Barnes, and V. Salomonson, "Terra and Aqua MODIS Thermal Emissive Bands On-orbit Calibration and Performance", IEEE Transactions on Geoscience and Remote Sensing, 53, 5709 - 5721, (2015).

[8] Madhavan, S., X. Xiong, A. Wu, B. N. Wenny, K. Chiang, N. Chen, Z. Wang, Y. Li, "Noise Characterization and Performance of MODIS Thermal Emissive Bands", IEEE Transactions on Geo science and Remote Sensing (10.1109/TGRS.2015.2514061), (2016).

[9] Sun, J., S. Madhavan, X. Xiong, and M. Wang, "Electronic crosstalk in Terra MODIS thermal emissive bands", Proc. SPIE 9607, 960730 (2015).

[10] Wang, Z., S. Madhavan, X. Xiong, A. Wu, and B.N. Wenny, "Monitoring and assessment of the temperature fluctuation of Aqua MODIS cold focal plane assembly", Proceedings of SPIE- Earth Observing Systems XVII, vol. 8510, issue 85100K, 2012. 